\title{
Modified Shuffled Frog Leaping Algorithm for Solving Economic Load Dispatch Problem
}

\author{
Priyanka Roy ${ }^{1}$, Abhijit Chakrabarti ${ }^{2}$ \\ ${ }^{1}$ Electrical engineering Department, Techno India, Kolkata, India \\ ${ }^{2}$ EE Department, Bengal Engineering and Science University, Shibpur, Howrah, India \\ E-mail:roy_priyan@rediffmail.com, a_chakrabarti55@yahoo.com \\ Received August 10, 2011; revised September 12, 2011; accepted September 24, 2011
}

\begin{abstract}
In the recent restructured power system scenario and complex market strategy, operation at absolute minimum cost is no longer the only criterion for dispatching electric power. The economic load dispatch (ELD) problem which accounts for minimization of both generations cost and power loss is itself a multiple conflicting objective function problem. In this paper, a modified shuffled frog-leaping algorithm (MSFLA), which is an improved version of memetic algorithm, is proposed for solving the ELD problem. It is a relatively new evolutionary method where local search is applied during the evolutionary cycle. The idea of memetic algorithm comes from memes, which unlike genes can adapt themselves. The performance of MSFLA has been shown more efficient than traditional evolutionary algorithms for such type of ELD problem. The application and validity of the proposed algorithm are demonstrated for IEEE 30 bus test system as well as a practical power network of 203 bus 264 lines 23 machines system.
\end{abstract}

Keywords: Economic Load Dispatch Modified Shuffled Frog Leaping Algorithm, Genetic Algorithm

\section{Introduction}

Economic load dispatch (ELD) is a familiar problem pertaining to the allocation of the amount of power to be generated by different units in the system on an optimum economic basis. The generated power has to meet the load demand and transmission losses. This implies that the dispatch at the true minimum cost requires that we take the network losses into account. Also for the secure operation of the power system, the generators must dispatch in such a way so that the transmission capacity limits are not exceeded.

Many researches are involved to tackle the ELD problem for significant economical benefit. Conventional methods such as lamda iteration method, gradient based method [1] are used to solve the ELD problem by changing the fuel cost curve in a piecewise linear function or monotonically increasing function. These methods ignore the portion of incremental cost curve that are not continuous or monotonically increasing. But input-output characteristics of modern units are inherently non-linear because of ramp rate limits, valve point loadings etc. So in classical method fuel cost curve is approximated according to their requirement but use of such approximation may lead to huge loss of revenue over the time. Dynamic programming, proposed in [2], is a method to solve non-linear and discontinuous ELD problem but with respect to system size, simulation time is increased rapidly in this method. Other than classical methods, different artificial intelligent based methods have been successfully utilized to compute ELD problem. These methods are evolutionary programming [3], particle swarm optimization [4], tabu search [5], differential evolution [6], biography based optimization [7], genetic algorithm [8], artificial neural network [9], intelligent water drop algorithm [10] etc. Each and every method has its own disadvantages. Neural network suffers from excessive iterations, resulting huge calculation as well as more processing time. Genetic algorithm has a disadvantage of premature convergence, and due to that its performance degrades and its search capability reduces. In PSO, the algorithm progresses slowly and due to its inability to adjust the velocity step size it may be difficult to continue the search at a finer grain. For multi modal function PSO sometimes fail to reach global optimal point. DE has been found to yield better and faster solution, satisfying all the constraints, both for uni-modal and multi-modal system by using its different crossover 
strategies. However with increase in system complexity and size, DE method is unable to map its entire unknown variables together, in an efficient way.

Recently, a new meta-heuristic algorithm called Shuffled Frog-Leaping Algorithm (SFLA) is introduced [11], it aims to model and mimic the behavior of frogs searching for food laid on stones randomly located in a pond. It combines the advantages of the genetic-based memetic algorithm (MA) and the social behavior-based Particle Swarm Optimization (PSO) algorithm and has found applications in areas such as optimizing bridge-deck repairs [12], materialized views selection [13], bi-criteria permutation flow shop scheduling problem [14], application to reservoir flood control operation [15] and a mixed-model assembly line sequencing problem [16].

This paper proposes a combined shuffled frog-leaping algorithm (SFLA) and a genetic algorithm (GA) that chooses genes (features) related to classification. It is named as modified shuffled frog-leaping algorithm (MSFLA) where two types of iterations (local and global search) are simultaneously performed to get better optimized value. In this method cross over operation has been implemented in both global and local iterations. Solving ELD problem using MSFLA technique is new and the application and validity of the proposed algorithm are demonstrated for IEEE 30 bus test system as well as for a practical power network of 203 bus 264 lines 23 machines eastern India grid system. It has been observed that compared to GA and common traditional method, MSFLA based ELD solutions yield better results from economic point of view.

A brief description and mathematical formulation of ELD problem has been discussed in the following section. The concept of SFLA is discussed in section III while the respective algorithm and parameter setting of MSFLA has been provided in section IV. Simulation studies are discussed in section $\mathrm{V}$ and conclusion is drawn in section VI.

\section{Nomenclature}

In the analytical model following symbols have been used:

$m$ and $n$ : Number of buses

$B_{i j}$ : Loss coefficients for active power

$\phi$ : Power factor angles of bus load

$\delta$ : Phase angles of bus voltages

$P_{D}$ : Real power demands

$P_{G}$ : Real power outputs

$P_{L}$ : Real loss .

$R_{i j}$ : Series resistance of lines

Suffix $i$ stands for $i^{\text {th }}$ bus while suffix $j$ stands for $j^{\text {th }}$ bus. The variables have been expressed in p.u. while the angles have been expressed in degree

\section{Economic Load Dispatch}

The aim of ELD is to optimize the cost function subjected to linear and non-linear equality and inequality constraints. The cost function $\left(F_{c_{\text {total }}}\right)$ of an N-bus power system having $N G$ number of fossil fuel units is given by

$$
F_{c_{\text {total }}}\left(=\sum_{i=1}^{N G} F_{c_{i}}\right)=\sum_{i=1}^{N G} \alpha_{i}\left(P_{G_{i}}\right)^{2}+\beta_{i} P_{G_{i}}+\gamma_{i}
$$

unit of cost/hr,

The active loss is conventionally expressed using B-coefficient (or loss coefficient) matrix and can be represented as [17],

$$
\begin{aligned}
P_{L} & =\sum_{i=1}^{n} \sum_{j=1}^{m} P_{G i} B_{i j} P_{G j} \\
& =B_{00}+\sum_{i=1}^{n} B_{i 0} P_{G i}+\sum_{i=1}^{n} \sum_{j=1}^{m} P_{G i} B_{i j} P_{G j}
\end{aligned}
$$

For a system of N-plants, the loss coefficients are given by [17]:

$$
\begin{gathered}
B_{i j}=\frac{\cos \left(\theta_{i}-\theta_{j}\right) R_{i j}}{\cos \phi_{i} \cos \phi_{j}\left|V_{i}\right|\left|V_{j}\right|} \\
B_{00}=\sum_{i=1}^{n} \sum_{j=1}^{m} P_{D i} B_{i j} P_{D j} \text { and } B_{i 0}=-\sum_{j=1}^{m}\left(B_{i j}+B_{j i}\right) P_{D j}
\end{gathered}
$$

\subsection{Power Balance}

The total generating power has to be equal to the sum of load demand and transmission-line loss:

$$
D+L-C<=O \text {, }
$$

where $D$ is total load, $L$ is transmission loss and $C$ is generated power.

The transmission loss can be represented by the Bcoefficient method as described in Equations (2) and (3).

\subsection{Maximum and Minimum Limits of Power}

The generation power of each generator has some limits and it can be expressed as

$$
P_{G_{i}}^{\min } \leq P_{G_{i}} \leq P_{G_{i}}^{\max }
$$

\section{Shuffled Frog Leaping Algorithm}

The shuffled frog-leaping algorithm (SFLA) is a heuristic search algorithms. It attempts to balance between a 
wide scan of a large solution space and also a deep search of promising location for a global optimum which can not be solved by traditional optimization techniques. It combines the benefits of a gene-based memetic algorithm (MA) and social behavior-based particle swarm optimization (PSO). MA is a gene-based optimization algorithm similar to a GA. In a GA, chromosomes are represented as a string consisting of a set of elements called "genes." Chromosomes in MA are represented by elements, called "memes." MA and GA differ in one aspect, i.e. MA implements a local search before crossover or mutations to determine offspring. After the local search, new offspring that obtains better results than original offspring, replaces original offspring and thus the evolutionary process continue. PSO is an evolutionary algorithm in which individual solutions are called "particle" (analogous to the GA chromosome), but PSO does not apply crossover and mutation to construct a new particle. Each particle changes its position and velocity based on the individual particle's optimal solution and the corporate optimal solution until a global optimal solution is found.

The SFLA is derived from a virtual population of frogs in which individual frogs are equivalent to the GA chromosomes and represent a set of solutions. Each frog is distributed to a different subset of the whole population, called a memeplex. An independent local search is conducted for each frog memplex and is called memeplex evolution. After a defined number of memetic evolutionary steps, frogs are shuffled among memeplexes enabling frogs to interchange messages among different memplexes. This ensures that they move to an optimal position similar to particles in PSO. Local search and shuffling continue until defined convergence criteria are met.

SFLA have demonstrated effectiveness in a number of global optimization problems which are difficult to solve using other method viz. intelligent water drop technique [10].The detail steps involved in SFLA is given as under.

\subsection{Initial Population}

An initial population of $P$ frogs is created randomly for a $S$-dimensional problem. A frog $i$ is represented by $\mathrm{S}$ variables,

$$
F_{1}=\left(f_{i 1}, f_{i 2}, f_{i 3}, \cdots\right)
$$

\subsection{Sorting and Distribution}

Frogs are sorted in descending order based on their fitness values. The entire population is then divided into $m$ memeplexes, each containing $n$ frogs (i.e., $P=m \times n$ ).

The first frog is distributed to the first memeplex, the second frog to the second, the $m$ frog to the $m$ memeplex, and the $m-1$ frog to the first memeplex and so on.

\subsection{Memeplex Evolution}

Within each memeplex, frogs with the best and the worst fitness are identified as $X_{b}$ and $X_{w}$, and the frog with the global best fitness is identified as $X_{g}$ separately. To improve upon the worst solution, an equation similar to PSO is used to update the worst solution, e.g., Equations (7) and (8):

Change in frog position

$$
D_{1}=\operatorname{rand}() \cdot X_{b}-X_{w}
$$

New position $X_{w}=$ current position $\left(X_{w}+D_{i}\right)$

$$
\left(D_{\max }>D>-D_{\max }\right)
$$

where rand() is a random number between 0 and 1 and Dmax is the maximum change allowed in a frog's position. If this process produces a better solution, it replaces the worst frog. If Equations (7) and (8) do not improve the worst solution, $X_{b}$ of Equation (7) is changed to $X_{g}$ and adapted to Equation (9).

Change in frog position

$$
\left(D_{1}\right)=\operatorname{rand}() \cdot X_{g}-X_{w}
$$

If Equations (7) and (9) do not improve the worst solution, then a new solution is randomly generated to replace that worst frog.

\subsection{Shuffling}

After a defined number of memeplex evolution steps, all frogs of memeplexes are collected, and sorted in descending order based on their fitness. Step 2 divides frogs into different memeplexes again and then step 3 is performed.

\subsection{Terminal Condition}

If a global solution or a fixed iteration number is reached, the algorithm stops.

\section{Programming Parameter and Algorithm}

In MSFLA programming a number of parameters need to be adjusted to compute best optimal value of the variables i.e., population size, number of memplexes, and number of global and local iteration.

1) Population size: it is a number of set of variables, defined as a total number of frogs. In this simulation procedure, population size has been taken as 100 . In- 
crease in number of population means good accuracy but it will lead to more propagation delay. After running the program with different number of population size, it has been observed that for this optimization problem, typically a population size of 100 is most suited for optimizing both processing time and value.

2) Number of memplexes: In this programming, number of memplexes is fixed at 10 . As population size and number of memplexes are user input, the given input of number of memplexes is such that there exists a certain number of frogs (population size/total number. of memplexes) in each memplexes.

3) Number of global iteration: In this type of iteration, the cross-over between best frog \& worst frog is done taking the whole population. One global iteration consists of local iterations as many as number of memplexes present. It is taken 10 here. Maximizing the number of global iteration gives more accurate results but it takes more time to process.

4) Number of local iteration: In this type of iteration, the cross-over between best frog \& worst frog is done in every single memplexes. Number of local iterations are taken as 20 here. Maximizing the number of local iterations also gives more accuracy but it gives more delay.

All the MSFLA parameters value discussed above is for IEEE 30 bus test system.

\section{Modified Shuffled Frog Leaping Algorithm}

Step 1: start

Step 2: population size (n), no. of memeplexes (m), number of local search within each memeplexes and number of global search are given as inputs.

Step 3: generate population of frogs $(F)$ randomly from the given data.

Step 4: evaluate fitness of $F$.

Step 5: sort F in descending order.

Step 6: cross-over between worst frog $\left(F_{w}\right)$ and best frog $\left(F_{b}\right)$ is done to get two new offsprings.

Step 7: replace $F_{b}$ and $F_{w}$ with two best frogs (according to their fitness) from four frogs(two parents and two offsprings).

Step 8: partition $\mathrm{F}$ into $\mathrm{m}$ memeplexes such that each memeplexes gets $(\mathrm{F} / \mathrm{m})$ frogs.

Step 9: find $F_{b}$ and $F_{w}$ from each memeplexes and do cross-over between them.

Step 10: get two new offspring from them and replace $F_{b}$ and $F_{w}$ with two best frogs(according to their fitness) from four frogs.

Step 11: check whether number of local search is completed or not, if not then go to step 9.

Step 12: if local search is completed then check whether number of global search is completed or not, if not then go to step 5 .

Step 13: if global iteration is completed then get the best solution (best fitness) from $F$.

Step 14: end.

It has been observed from the above algorithm that proposed MSFLA performs two simultaneous crossover, i.e., global (Step 6 and 7) and local (Step 9 and 10) search to produce new offspring which gives better result compared to SFLA.

\section{Simulation}

To examine the validity of MSFLA model for the ELD problem, IEEE 30 bus test system and a practical power network of 203 bus 264 lines 23 machines system have been considered. The result of proposed MSFLA model has been compared with GA based ELD result and classical iteration method. A reasonable B-loss coefficient matrix of the system has been employed to calculate transmission loss. The detail calculation part of ELD problem is concentrated on IEEE 30 bus test system followed by the power scheduling of practical system. The test system and production units' properties are given in Tables 1 and 2 for IEEE 30 bus system.

Table 3 shows different parameters of ELD scheduling of IEEE 30 bus test system with three computational techniques. It has been shown that compared to GA and classical method, MSFLA technique in respect to cost and power scheduling is better than the other two methods, though the computational time is more in MSFLA rather than GA. In MSFLA, cross over operation is performed in local as well as global iteration time where as in GA there is only one cross over operation. With respect to transmission loss, MSFLA computation yields

Table 1. Test system properties.

\begin{tabular}{ll}
\hline Number of buses & 30 \\
\hline Number of generator units & 6 \\
Number of branches & 43 \\
Number of tie lines & 6 \\
\hline
\end{tabular}

Table 2. Production units’ properties.

\begin{tabular}{cccccc}
\hline \multirow{2}{*}{$\begin{array}{c}\text { Generator } \\
\text { No }\end{array}$} & $\boldsymbol{P}_{\max (\mathbf{M W})}$ & $\boldsymbol{P}_{\min (\mathbf{M W})}$ & \multicolumn{3}{c}{ Cost Co-efficient } \\
\cline { 4 - 6 } & & & $\alpha_{i}$ & $\beta_{i}$ & $\gamma_{i}$ \\
\hline 1 & 145.5 & 120.5 & 0.074 & 1.083 & 25 \\
2 & 70.6 & 50.6 & 0.089 & 1.033 & 24 \\
3 & 35.6 & 20.4 & 0.089 & 1.033 & 22 \\
4 & 50 & 30 & 0.074 & 1.083 & 21 \\
5 & 25.9 & 10.8 & 0.089 & 1.033 & 23 \\
6 & 25.9 & 10.8 & 0.053 & 1.17 & 29 \\
\hline
\end{tabular}


Table 3. Computation of best outputs of 6 units systems using different method.

\begin{tabular}{|c|c|c|c|c|}
\hline \multicolumn{2}{|c|}{ Item (p.u. values) } & \multirow{2}{*}{$\begin{array}{c}\text { CLASSICAL } \\
\text { METHOD }\end{array}$} & \multirow{2}{*}{$\begin{array}{c}\text { GA } \\
1.385\end{array}$} & \multirow{2}{*}{$\begin{array}{c}\text { MSFLA } \\
1.391\end{array}$} \\
\hline \multirow{6}{*}{$\begin{array}{c}\text { Generating } \\
\text { power of } 6 \\
\text { generating } \\
\text { unit }\end{array}$} & $\mathrm{P} 1$ & & & \\
\hline & $\mathrm{P} 2$ & 0.5756 & 0.576 & 0.533 \\
\hline & P3 & 0.2456 & 0.246 & 0.237 \\
\hline & $\mathrm{P} 4$ & 0.35 & 0.35 & 0.368 \\
\hline & P5 & 0.179 & 0.178 & 0.159 \\
\hline & P6 & 0.1689 & 0.169 & 0.144 \\
\hline \multicolumn{2}{|c|}{ Total power (pu) } & 2.9039 & 2.904 & 2.832 \\
\hline \multicolumn{2}{|c|}{ Active loss (pu) } & 0.065 & 0.071 & 0.06 \\
\hline \multicolumn{2}{|c|}{ Cost of power (INR/hr) } & 147.2998 & 147.3 & 147.220 \\
\hline \multicolumn{2}{|c|}{ Processing time (Sec) } & 0.035 & 0.008 & 0.009 \\
\hline
\end{tabular}

lower loss compared to classical method and GA.

In Table 4, another simulation result has been shown where a practical power system having 203 buses, 264 lines, 23 machines of eastern grid of India is simulated using MSFLA, GA and classical method.

It has been observed from Table 4 that in a large system MSFLA based ELD scheduling still shows better result compared to GA and classical method. The total power generated using MSFLA being lesser in comparison to compute power by other two methods, it is most logical that MSFLA technique provides a tool for reduction in total cost of generated power.

The advantages of MSFLA over the other methods are discussed below:

1) In MSFLA, two types of iterations (local and global search) are simultaneously performed to get better optimized value whereas in GA there is only global search. MSFLA has the ability to reach global minima in a consistent manner with better convergence characteristic. In case of classical method only global search is performed.

2) In case of memory usage and time complexity, it has been observed from table III that MSFLA based solution takes more CPU time compared to GA based solution as two simultaneous iterations (global and local) are computed in MSFLA whereas GA based programming has been performed with single iteration. But in case of minimizing generation cost which is the main objective of ELD problem, MSFLA shows better result compared to GA and any other traditional method. More over transmission losses are also reduced using this method.

\section{Conclusions}

In this paper economic load dispatch problem has been
Table 4. Computation of best outputs of 23 units system using different method.

\begin{tabular}{|c|c|c|c|}
\hline $\begin{array}{l}\text { Power Generation } \\
\text { Of each unit } \\
\text { (p.u. values) }\end{array}$ & $\begin{array}{l}\text { CLASSICAL } \\
\text { METHOD }\end{array}$ & GA & MSFLA \\
\hline 1 & 5.4 & 5.48226 & 5.38 \\
\hline 2 & 5.4 & 5.49353 & 5.4 \\
\hline 3 & 3.8 & 3.48685 & 3.332 \\
\hline 4 & 5.4 & 5.50104 & 5.456 \\
\hline 5 & 3.8 & 3.49652 & 3.75 \\
\hline 6 & 2.4 & 2.40876 & 2.5 \\
\hline 7 & 0.9 & 0.89 & 0.8 \\
\hline 8 & 0.46 & 0.40835 & 0.456 \\
\hline 9 & 1.8 & 1.85684 & 1.79 \\
\hline 10 & 2 & 2.18023 & 2.05 \\
\hline 11 & 1.8 & 1.88354 & 1.889 \\
\hline 12 & 2.7 & 2.83909 & 2.56 \\
\hline 13 & 0.243 & 0.241 & 0.251 \\
\hline 14 & 0.108 & 0.1 & 0.1005 \\
\hline 15 & 0.54 & 0.51197 & 0.495 \\
\hline 16 & 6 & 6.09554 & 6.01 \\
\hline 17 & 0.5 & 0.54414 & 0.49 \\
\hline 18 & 1.5 & 1.55 & 1.64 \\
\hline 19 & 6 & 6.08307 & 6.07 \\
\hline 20 & 0.204 & 0.2 & 0.23 \\
\hline 21 & 0.2 & 0.2 & 0.21 \\
\hline 22 & 0.22 & 0.2 & 0.2 \\
\hline 23 & 0.4 & 0.39 & 0.41 \\
\hline Total Power (pu) & 51.775 & 52.04273 & 51.4695 \\
\hline Active loss (pu) & 1.4452 & 1.4 & 1.387 \\
\hline Processing time (sec) & 1.2 & 0.8 & 1.1 \\
\hline Cost of power (INR/hr) & 1318.1 & 1317.89 & 1316.78 \\
\hline
\end{tabular}

formulated as a multi objective problem to optimize fuel cost as well as to minimize system loss. This problem is solved using MSFLA, GA and classical lamda iteration method. Using IEEE 30 bus test system as a standard system and a practical power system of 203 bus 264 lines 23 machines, it has been observed that MSFLA method is more efficient than other programming techniques. The good performance of MSFLA in ELD problem discussed in this paper provides some evidence that 
MSFLA theory can be successfully applied to various practical power system optimization problems in the future.

\section{Acknowledgements}

The authors would like to thank Mr. Pritam Roy, Computer science department, Govt. College of ceramic technology, for his relevant support related to the MSFLA programming.

\section{References}

[1] A. El-Keib, H. Ma and J. L. Hart, "Environmentally Constrained Economic Dispatch using the Lagrangian Relaxation Method," IEEE Transactions on Power System, Vol. 9, No. 4, 1994, pp. 1723-1729. doi:10.1109/59.331423

[2] Z. X. Liang and J. D. Glover, "A Zoom Feature for a Dynamic Programming Solution to Economic Dispatch including Transmission Losses," IEEE Transactions on Power Systems, Vol. 7, No. 2, 1992, pp. 544-550. doi:10.1109/59.141757

[3] T. Jayabarathi, G. Sadasivam and V. Ramachandran, "Evolutionary Programming based Economic dispatch of Generators with Prohibited Operating Zones," Electrical Power System Research, Vol. 52, No. 3, 1999, pp. 261266. doi:10.1016/S0378-7796(99)00025-5

[4] J. B. Park, K. S. Lee, J. R. Shin and K. Y. Lee, "A Particle Swarm Optimization for Economic Dispatch with Non Smooth Cost Functions," IEEE Transaction on Power Systems, Vol. 8, No. 3, 1993, pp. 1325-1332.

[5] W. M. Lin, F. S. Cheng and M. T. Tsay, "An Improved Tabu Search for Economic Dispatch with Multiple Minima," IEEE Transaction on Power Systems, Vol. 17, No. 1, 2002, pp. 108-112. doi:10.1109/59.982200

[6] N. Nomana and H. Iba, "Differential Evolution for Economic Load Dispatch Problems," Electric Power Systems Research, Vol. 78, No. 8, 2008, pp. 1322-1331. doi:10.1016/j.epsr.2007.11.007

[7] A. Bhattacharya and P. K. Chattopadhyay, "BiogeographyBased Optimization for Different Economic Load Dispatch Problems," IEEE Transactions on Power Systems, Vol. 25, No. 2, 2010, pp. 1064-1077. doi:10.1109/TPWRS.2009.2034525
[8] P. H. Chen and H. C. Chang, "Large-Scale Economic Dispatch by Genetic Algorithm,” IEEE Transactions on Power System, Vol. 10, No. 4, 1995, pp. 1919-1926. doi:10.1109/59.476058

[9] C.-T. Su and C.-T. Lin, "New Approach with a Hopfield Modeling Framework to Economic Dispatch," IEEE Transaction on Power System, Vol. 15, No. 2, 2000, pp. 541-545. doi:10.1109/59.867138

[10] S. R. Rayapudi, "An Intelligent Water Drop Algorithm for Solving Economic Load Dispatch Problem,” International Journal of Electrical and Electronics Engineering, Vol. 5, No. 2, 2011, pp. 43-49.

[11] M. M. Eusuff and K. E. Lansey, "Optimization of Water Distribution Network Design Using the Shuffled Frog Leaping Algorithm," Journal of Water Resources Planning and Management, Vol. 129, No. 3, 2003, pp. 210225. doi:10.1061/(ASCE)0733-9496(2003)129:3(210)

[12] E. Elbeltagi, “A Modified Shuffled-Frog-Leaping Algorithm for Optimizing Bridge-Deck Repairs," International Conference on Bridge Management System Monitaring, Assessment and Rehabilitation System, Cairo, 2123 March 2006.

[13] X. Li, X. Qian, J. L. Jiang and Z. Q. Wang, "Shuffled Frog Leaping Algorithm for Materialized Views Selection," 2010 Second International Workshop on Education Technology and Computer Science, Wuhan, 6-7 March 2010, pp. 7-10. doi:10.1109/ETCS.2010.480

[14] A. Rahimi-Vahed and A. H. Mirzaei, "Solving a Bi-Criteria Permutation Flow-Shop Problem using Shuffled Frog-Leaping Algorithm," The International Journal of Soft Computing-A Fusion of Foundations, Methodologies and Applications , Vol. 12, No. 5, 2010, pp. 435-452.

[15] Y. H. Li, J. Z. Zhou, Y. C. Zhang, H. Qin and L. Liu, "Novel Multiobjective Shuffled Frog Leaping Algorithm with Application to Reservoir Flood Control Operation," Journal of Water Resource Planning and Management, Vol. 136, No. 2, 2010, pp. 217-227.

[16] A. Rahimi-Vahed and A. H. Mirzaei, "A Hybrid Multi-Objective Shuffled Frog-Leaping Algorithm for a Mixed-Model Assembly Line Sequencing Problem,” Journal of Computers and Industrial Engineering, Vol. 53, No 4, 2007.

[17] A. Chakrabarti and S. Halder, "Power System Analysis, Operation and Control,” 3rd Edition, PHI Learning Private Ltd., New Delhi, 2010. 